\title{
Tratamiento del trombo graso en la vena renal con extensión a la vena cava: reporte de dos casos
}

\section{Treatment of a fatty tumor thrombus in the renal vein extending into the vena cava: Presentation of two cases}

\author{
Marta Antón-Juanilla, Antonio Arruza-Echeverría
}

\begin{abstract}
Resumen
ANTECEDENTES: Los trombos grasos en la vena renal son raros y el angiomiolipoma es la única tumoración benigna que puede asociarlos.

CASOS CLínICOS: Caso 1: paciente femenina de 70 años, con hallazgo durante el seguimiento endocrino de un trombo graso en la vena renal izquierda, con extensión a la vena cava sin clara lesión renal asociada. Se trató de manera conservadora con anticoagulantes y estudios de control anual. Caso 2: paciente femenina de 43 años, con diagnóstico durante el embarazo de una masa en el seno renal derecho, con trombo graso en la vena renal y cava. Se le indicó un anticoagulante y en el posparto se le efectuó nefrectomía radical derecha con trombectomía. El diagnóstico anatomopatológico fue angiomiolipoma.

CONCLUSIONES: La coexistencia de un trombo graso en la vena renal es poco común y suele estar ligada a un angiomiolipoma renal. Por el riesgo de embolismo pulmonar o diferenciación maligna está indicado el tratamiento quirúrgico con nefrectomía radical y trombectomía, incluso en pacientes asintomáticos. En el caso de un trombo graso aislado en pacientes con comorbilidades, el tratamiento conservador con anticoagulación es una opción válida.
\end{abstract}

PALABRAS CLAVE: Trombo; vena renal; angiomiolipoma; anticoagulantes; vena cava; embolismo.

\section{Abstract}

BACKGROUND: A fatty tumor thrombus in the renal vein is rare and angiomyolipoma is the only benign tumor associated with it.

CLINICAL CASES: Case 1: a 70-year-old woman presented with the incidental finding of a fatty tumor thrombus, during endocrine follow-up, in the left renal vein that extended into the vena cava, with no clearly associated kidney lesion. She was managed conservatively with anticoagulation and yearly control. Case 2: a 43-year-old woman was diagnosed during pregnancy with a mass in the right renal sinus, with a fatty thrombus in the renal vein and vena cava. Anticoagulation was begun and in the postpartum period, she underwent a right radical nephrectomy with thrombectomy. The anatomopathologic diagnosis was angiomyolipoma.

CONCLUSIONS: A fatty tumor thrombus in the renal vein is rare and usually linked to a renal angiomyolipoma. Due to the risk for pulmonary embolism or malignant differentiation, radical nephrectomy with thrombectomy is the indicated treatment, even in asymptomatic patients. In the case of an isolated fatty thrombus in patients with comorbidities, conservative treatment is a valid option.

KEYWORDS: Thrombus; Renal vein; Angiomyolipoma; Anticoagulants; Vena cava; Embolism.
Hospital Universitario Cruces, España.

Recibido: abril 2018

Aceptado: agosto 2018

Correspondencia Marta Antón Juanilla m_curie91@hotmail.com

Este artículo debe citarse como Antón-Juanilla M, Arruza-Echeverría A. Tratamiento del trombo graso en la vena renal con extensión a la vena cava: reporte de dos casos. Rev Mex Urol. 2018 sept-oct;78(5):379-384.

Doi: https://doi.org/10.24245/revmexurol.v78i5.2170 


\section{ANTECEDENTES}

La coexistencia de un trombo graso en la vena renal es realmente excepcional, incluso asociada con un angiomiolipoma; solo se han reportado poco menos de un centenar de casos. Su aparición implica la posibilidad de complicación por el riesgo de embolismo; el tratamiento casi siempre es nefrectomía con cavotomía.

El angiomiolipoma renal es la única tumoración benigna que puede evolucionar a un trombo en la vena cava. Se identifican esporádicamente (80\%) en adultos (edad media 43 años) con mayor predominio en mujeres $(4: 1)$ y en pacientes con facomatosis $(20 \%)$, principalmente esclerosis tuberosa, y casi siempre se describen como lesiones sólidas compuestas de vasos distróficos, células musculares lisas y tejido adiposo. ${ }^{1}$

Las indicaciones de tratamiento incluyen: riesgo de sangrado espontáneo en lesiones mayores de $4 \mathrm{~cm}$, sospecha de lesión maligna y riesgo de insuficiencia renal. Se dispone de distintas opciones terapéuticas: embolización, inhibidores mTOR, como el everolimus y la cirugía. ${ }^{2}$

Enseguida se reportan dos casos con trombo graso en la vena renal, con diferentes características que dieron lugar a tratamientos clínicos opuestos.

\section{CASOS CLÍNICOS}

Caso 1

Paciente femenina de 70 años, con antecedentes de suprarrenalectomía izquierda por metanefrinas discretamente elevadas y esplenectomía en el mismo tiempo quirúrgico por linfoma esplénico; permanece en controles anuales con tomografía computada en el servicio de Endocrinología. En uno de esos controles se diagnosticó un trombo graso en la vena renal izquierda.
En la tomografía se describió la vena renal izquierda aumentada de tamaño y ocupada en su luz por tejido de densidad grasa $(-100 \mathrm{UH}) \mathrm{de}$ alrededor de $9 \mathrm{~cm}$ de longitud hasta la desembocadura de la vena cava inferior, con impronta en el interior de ésta. No se visualizó el angiomiolipoma renal que sugiriera la extensión hacia la vena. Figura 1

La resonancia magnética, indicada para valorar la posibilidad de origen renal, mostró al riñón izquierdo con morfología normal, grosor cortical conservado y sin lesiones focales.

Ante la imagen de densidad grasa intravascular que se extendía desde el hilio renal hasta la vena cava y que no condicionaba estenosis con dilatación previa ni afectación renal, se acordó guardar una actitud conservadora y controles anuales.

En la revisión del cuarto año se objetivó aumento de tamaño del trombo graso respecto de las imágenes previas; ocupaba prácticamente la totalidad de su luz, aunque con permeabilidad de la vena en torno al trombo y con extensión desde

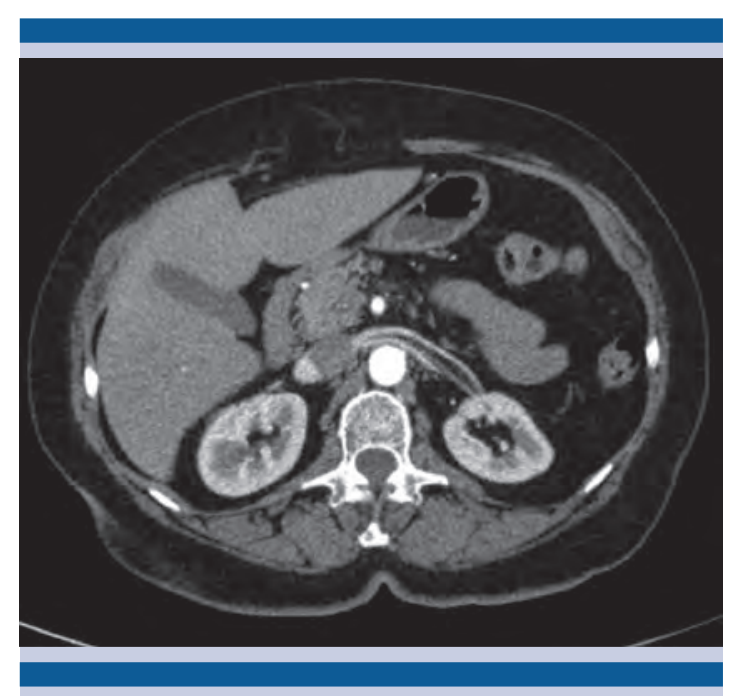

Figura 1. Tomografía axial computada con trombo graso en la vena renal izquierda. 
el polo superior renal hasta la vena cava inferior hepática. Continuó sin poder identificarse una clara lesión en el parénquima renal izquierdo, aunque con un área de mayor comportamiento graso en el polo renal superior. Figura 2

En una sesión multidisciplinaria que tomó en cuenta el diagnóstico reciente en la paciente con carcinoma ductal infiltrante de mama y ausencia de afectación renal, se decidió mantener una conducta conservadora, con anticoagulación y seguimiento anual.

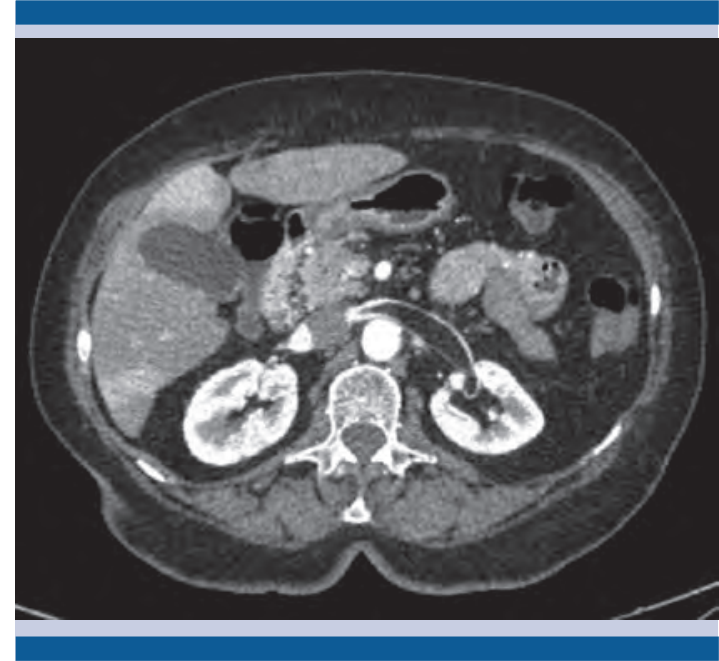

Figura 2. Tomografía axial computada con evolución del trombo graso en la vena renal izquierda.

\section{Caso 2}

Paciente femenina de 43 años, sin antecedentes médicos de interés, valorada por el urólogo ante el hallazgo de un trombo en la vena renal derecha hacia la cava inferior. Durante el embarazo, que cursó con diabetes gestacional, tuvo elevación de transaminasas; debido a esto se indicó una ecografía abdominopélvica en la que llamó la atención una zona hiperecogénica de localización medular pericalicial que se extendía cranealmente hacia la vena renal derecha, con extensión a la vena cava inferior (Figura 3). La

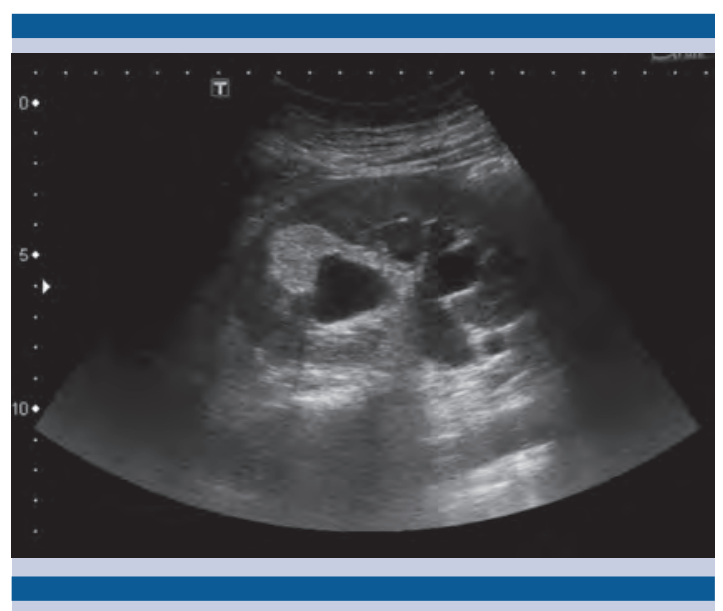

Figura 3. Ecografía renal con área hiperecogénica medular.

paciente no había tenido molestias lumbares ni otros síntomas previos en posible correlación con los hallazgos.

Con el propósito de valorar mejor esos hallazgos se indicó una resonancia magnética (Figura 4), que describió la ocupación y distensión de la totalidad de la vena renal derecha que se extendía por la vena cava inferior suprarrenal e infrahepática, datos sugerentes de trombosis. También se encontró tejido isointenso en torno al trombo que rodeaba al grupo calicial en el in-

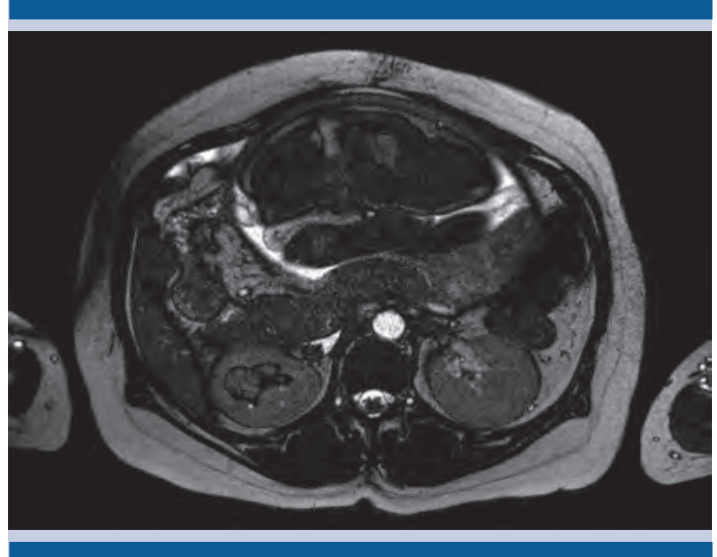

Figura 4. Resonancia magnética con trombo graso en la vena renal derecha. 
fundíbulo superior derecho; pudo corresponder con una extensión de la trombosis en las venas intersticiales parenquimatosas.

Con el diagnóstico de trombo graso en la vena renal, con extensión a la vena cava, la paciente ingresó a las 37 semanas de embarazo al servicio de Obstetricia. En una sesión multidisciplinaria se decidió iniciar la anticoagulación a dosis terapéutica; se desestimó la colocación de un filtro en la vena cava.

A las 38 semanas de embarazo se produjo el parto vaginal, con ventosa, sin contratiempos. Durante el puerperio se practicó angio-TAC para revalorar la trombosis de la vena renal derecha. En el seno renal que rodea al sistema infundíbulo calicial del grupo superior se visualizó una masa de aproximadamente $45 \times 28 \mathrm{~mm}$ de naturaleza grasa $(-50 \mathrm{UH})$ que se continuaba con un trombo que ocupaba la luz de la vena renal derecha y se extendía craneocaudalmente $6 \mathrm{~cm}$ en la vena cava infrahepática. Figura 5

Los hallazgos se interpretaron como tumor renal, tipo angiomiolipoma que infiltraba la vena renal y cava inferior. Ante el riesgo de evolución y libe-

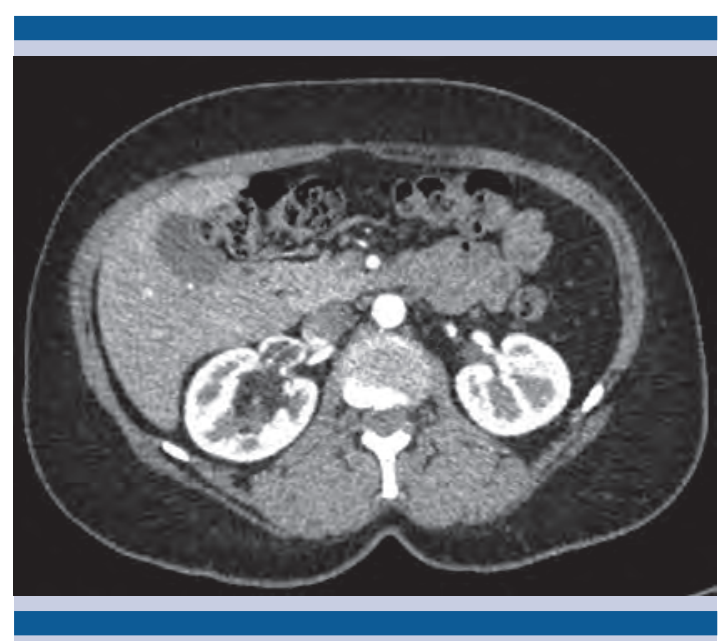

Figura 5. AngioTAC con masa en el seno renal derecho. ración de los émbolos se optó por la nefrectomía derecha con trombectomía. Por la alta complejidad que planteaban las características del tumor no se vislumbró la posibilidad de practicar una cirugía parcial en el riñón.

Con la paciente en decúbito supino se utilizó como vía de acceso una incisión de Chevron que permitió acceder hasta el peritoneo, rechazar las asas intestinales y mediante la maniobra de Kocher exponer la vena cava. Se liberó el riñón derecho, preservó la glándula suprarrenal y se ligó la arteria renal con tres hem-o-lok. En el uréter se utilizó ligasure. Enseguida se referenció la vena cava en su porción infrarrenal y en el límite superior del trombo. Se ligaron las venas renales derechas con Endo-GIA vascular, previo pinzamiento de la vena cava. Después se efectuó una cavotomía que permitió la extracción del trombo de aspecto graso. Se cerró con sutura continua de prolene y se procedió a revisar exhaustivamente la hemostasia. Para mejor control se dejó surgicel fibrilar y Tachosil en el área quirúrgica.

El sangrado intraoperatorio fue de 500 cc y la paciente fue dada de alta a los 11 días posquirúrgicos, sin complicaciones.

En el análisis anatomopatológico de la pieza quirúrgica se observó una tumoración amarillenta que protruía la zona del hilio renal, sin revestimiento por cápsula. Se expandía hacia el parénquima, adelgazaba la corteza sin rebasar la cápsula perirrenal ni infiltrar la vena renal. En la vena cava había un trombo. El diagnóstico final fue angiomiolipoma de predominio adiposo.

\section{DISCUSIÓN}

El angiomiolipoma es un tumor benigno que casi siempre se diagnostica de manera fortuita y no tiene mayores consecuencias. En ocasiones, debido al tamaño o localización puede convertirse en un padecimiento grave. Se trata del segundo 
tumor renal con mayor incidencia (23\%) durante el embarazo, seguido del carcinoma de células claras $^{4}$ que puede complicarse, de manera excepcional, con la coexistencia de un trombo en la vena cava. El crecimiento del tumor se afecta con el embarazo, sobre todo durante el segundo trimestre debido a la progesterona. ${ }^{5}$ En pacientes con diagnóstico previo se recomienda la monitorización ecográfica periódica, en algunos casos se prefiere el tratamiento quirúrgico antes del embarazo, para evitar complicaciones hemorrágicas.

El tratamiento del angiomiolipoma con trombo graso es complejo y requiere la participación de un grupo multidisciplinario que incluya al urólogo, al cirujano vascular y al obstetra durante el embarazo. El tratamiento conservador con anticoagulación es necesario para prevenir la extensión del trombo y la formación de émbolos. Cuando el angiomiolipoma con trombo representa una amenaza para la vida de la paciente, por el riesgo hormonal de rápida expansión o el tromboembolismo pulmonar, durante el embarazo puede optarse por la nefrectomía con cavotomía. $^{6}$

Con el propósito de disminuir el riesgo de embolismo pulmonar, algunos cirujanos recomiendan la implantación de un filtro de vena cava antes de la cirugía. En los casos en que sea posible, porque la extensión del trombo no alcanza las venas suprahepáticas, esta medida se asocia con mayor riesgo de trombosis e inflamación. Además, el empleo de un filtro permanente puede ocasionar edema en las extremidades inferiores, infección e incluso insuficiencia orgánica. ${ }^{7}$ En los pacientes sin filtro se debe ser extremadamente cuidadoso durante la movilización renal evitando presiones que puedan liberar émbolos.

En los casos aquí reportados se eligieron distintas opciones terapéuticas que dependieron de las características particulares de las pacientes.
El tratamiento conservador del trombo graso es posible cuando se limita a una extensión vascular infrahepática, sin identificarse una lesión renal que pueda justificar la nefrectomía. Además, en este caso se añadió un diagnóstico oncológico durante el seguimiento, que sumaba comorbilidad pero se mantenía la actitud expectante que se reevaluó en los sucesivos controles. En pacientes jóvenes con diagnóstico por imagen de angiomiolipoma con extensión del trombo hasta la cava es probable un crecimiento tumoral por la exposición hormonal; en estos casos debe proponerse una cirugía radical y efectuarla en el momento obstétrico más adecuado, con el propósito de evitar sangrados espontáneos, embolismos o malignidad de la lesión renal.

\section{CONCLUSIONES}

La coexistencia de un trombo graso en la vena renal es poco común y suele estar ligada a un angiomiolipoma renal. Por el riesgo de embolismo pulmonar o diferenciación maligna está indicado el tratamiento quirúrgico con nefrectomía radical y trombectomía, incluso en pacientes asintomáticos. En el caso de un trombo graso aislado en pacientes con comorbilidades, el tratamiento conservador con anticoagulación es una opción válida.

\section{REFERENCIAS}

1. Fittschen A, et al. Prevalence of sporadic renal angiomyolipoma: a retrospective analysis of 61,389 in- and outpatients. Abdom Imaging 2014;39(5):1009-13. https://doi. org/10.1007/s00261-014-0129-6

2. Seyam RM, et al. The risks of renal angiomyolipoma: reviewing the evidence. J Kidney Cancer VHL 2017; 4(4):1325. http://dx.doi.org/10.15586/jkcvhl.2017.97

3. Kutikov A, et al. The R.E.N.A.L. Nephrometry Score: A comprehensive standardized system for quantitating renal tumor size, location and depth. J Urol. 2009; 182:844-53. https://doi.org/10.1016/j.juro.2009.05.035

4. Raft J, et al. Influence of pregnancy on renal angiomyolipoma. Gynecol Obstet Fertil 2005; 33:898-906. https://doi. org/10.1016/j.gyobfe.2005.09.015 
5. Rothberg G, et al. Rapid growth of a kidney angiomyolipoma after initiation of oral contraceptive therapy. Obstet Gynecol 2006; 108:734-6. doi: 10.1097/01. AOG.0000188062.13749.ef

6. Lopater J, et al. Management of angiomyolipoma vena cava thrombus during pregnancy. Obstet Gyne- col. 2011; February;117(2 Pt 2):440-3. doi: 10.1097/ AOG.0b013e3181edbc56

7. Xiang L, et al. Malignant epithelioid angiomyolipoma invading the inferior vena cava: using a temporary vena cava filter to prevent tumour emboli during nephrectomy. Can Urol Assoc J. 2014 Jul-Aug; 8(7-8): E564-E566. http:// dx.doi.org/10.5489/cuaj.1814

\section{AVISO IMPORTANTE}

La Revista Mexicana de Urología se convierte en una publicación solo digital, con todas las ventajas que los medios y dispositivos electrónicos ofrecen. Usted podrá revisar la información mediante el sitio web (www.revistamexicanadeurologia.org.mx) o descargando la app para Android o iPhone.

Para consultar el texto completo de los artículos deberá registrarse por una sola vez con su correo electrónico, crear una contraseña, indicar su nombre, apellidos y especialidad.

Esta información es indispensable para saber qué consulta y cuáles son sus intereses, y poder en el futuro inmediato satisfacer sus necesidades de información. 